\title{
Correlation of Stone Attenuation Measurement on Non- Contrast Enhanced Computed Tomography with Stone Fragmentation Using Extracorporeal Shock Wave Lithotripsy in Upper Urinary Calculi
}

\author{
Kamal Sharma , P Satish Kumar², Ranjana Gupta ${ }^{3}$, Puneet Mittal ${ }^{4}$ \\ ${ }^{1}$ Associate Professor Department of Urology, Maharishi Markandeshwar Institute of Medical Sciences and Research, Mullana, \\ Ambala, Haryana, ${ }^{2}$ Senior Consultant, Department of Urology, Sri Satya Sai Institute of Higher Medical Sciences, Puttaparthy, \\ Andhra Pradesh, ${ }^{3}$ Associate Professor, Department of Radiodiagnosis, Maharishi Markandeshwar Institute of Medical \\ Sciences and Research, Mullana, Ambala, ${ }^{4}$ Associate Professor, Department of Radiodiagnosis, Maharishi Markandeshwar \\ Institute of Medical Sciences and Research, Mullana, Ambala, India
}

Corresponding author: Ranjana Gupta, F-9, MM University, Mullana, Ambala, Haryana, India

DOI: $10.21276 /$ ijcmsr.2018.3.2.20

How to cite this article: Kamal Sharma, P Satish Kumar, Ranjana Gupta, Puneet Mittal. Correlation of stone attenuation measurement on non-contrast enhanced computed tomography with stone fragmentation using extracorporeal shock wave lithotripsy in upper urinary calculi. International Journal of Contemporary Medicine Surgery and Radiology. 2018;3(2):B81-B84.

\section{A B S T R A C T}

Introduction: Extracorporeal shock wave lithotripsy (ESWL) has been extensively used in management of urinary calculi, however its success rate is variable depending upon the size and attenuation value of stones. Non-contrast computed tomography (NCCT) can accurately measure these parameters. The purpose of present study was to assess role of NCCT Hounsfield unit (HU) measurement in upper urinary calculi for predicting success rate of ESWL.

Material and methods: The present prospective study was conducted in 30 subjects with symptomatic radio-opaque solitary renal or upper ureter calculus measuring $5-20 \mathrm{~mm}$. Patients were divided into two groups (A and B), depending on the CT attenuation values of less or more than $750 \mathrm{HUs}$ respectively. All treatment was done by an electromagnetic lithotripter (Dornier Compact Delta, Singapore) under fluoroscopic guidance. Cases of residual fragments larger than $5 \mathrm{~mm}$ or of a stone that did not fragment satisfactorily were considered failures. Mean HU per stone was compared in the stone free and residual stone groups.

Results: Out of 30 subjects, 17 belonged to group A (stone $\mathrm{HU}$ value $<750 \mathrm{HU}$ ) and 13 belonged to group $\mathrm{B}$ (stone attenuation value $\geq 750 \mathrm{HU}$ ). The mean number of shock waves delivered in two groups A and B were 4411.76 and 7615.38 respectively $(p<0.0001)$. Mean stone size in group A was $1.51 \mathrm{~cm}$ and that in group B was 1.59 ( $p$ value- 0.5346$)$. The stone clearance rate in Group A was $100 \%$ versus Group B where it was only $15.4 \%(p<0.001)$. Overall, mean attenuation values for the stonefree and residual stone groups were significantly different ( 514.10 versus $970.36 \mathrm{HU}$, respectively, $\mathrm{p}<0.0001$ ).

Conclusion: NCCT attenuation value has inverse relationship with ESWL outcome with poor response at $\mathrm{HU}$ value $\geq 750 \mathrm{HU}$.

Key words: Computed Tomography, ESWL, Hounsfield Unit, Urinary Calculi

\section{INTRODUCTION}

At its inception in the early 1980s, shock wave lithotripsy was viewed as the ideal minimally invasive therapy for renal calculi. ${ }^{1}$ The side effects were minimal and the treatment was believed to be highly effective. It largely swept open lithotomy and even today shockwave lithotripsy (SWL) is the most commonly utilized modality for urolithiasis. Nonetheless, it is evident that SWL is not ideal for all stones.

The ability to identify appropriate candidates for SWL therefore continues to be refined. Advancements in imaging have significantly contributed to this process. In the mid1990s, computed tomography (CT) began to replace intravenous urography (IVU), abdominal films (KUB), and ultrasound (US) in stone diagnosis. Studies demonstrated that CT had superior sensitivity and specificity for stone diagnosis compared to the aforementioned modalities. Now non-contrast CT (NCCT) is the gold standard for detection of urinary system calculi. ${ }^{2}$

CT is also clinically useful as it is able to show alternate renal and non-renal pathology if present, and also can detect uric acid stones which otherwise may not be detected on KUB films. ${ }^{3}$ In the last decade, the utility of CT has gone beyond simple diagnosis. By determining stone attenuation, NCCT can give some idea of composition of stones with uric acid stones being of lower attenuation (200-400HU) and calcium phosphate and calcium oxalate monohydrate stones being of high attenuation $(>1000 \mathrm{HU})$. The success rate of ESWL also depends upto stone hardness/fragility which is related 
to its density and composition. NCCT determination of attenuation value is helpful and high attenuation correlates with more density. ${ }^{4}$ We evaluated the role of NCCT for predicting success rate of ESWL.

\section{MATERIAL AND METHODS}

The present prospective study of ESWL outcome (in terms of stone fragmentation) by measuring the calculus density (in Hounsfield units) using non-contrast enhanced CT (NCCT) scan was conducted in Department of Urology, Sri Satya Sai Institute of higher Medical Sciences, Puttaparthy, Andhra Pradesh.

A total of 21 men and 9 women (15-60 years) participated in this prospective study conducted between March 20th 2009 to 31st March 2011. The study was approved by institutional ethics committee. Patients with a symptomatic radio-opaque solitary renal or upper ureter calculus larger than 5 and up to $20 \mathrm{~mm}$. in the largest dimension in satisfactorily functioning and unobstructed renal units were included in the study.

Those with a calculus less than 5 and greater than 20 $\mathrm{mm}$, elevated serum creatinine (greater than $1.5 \mathrm{mg} / \mathrm{dl}$ ), bleeding diathesis, solitary functioning kidney, stents in situ, developing steinstrasse during therapy were excluded from study. The maximal linear diameter of the calculus was measured by plain $\mathrm{x}$-ray of the kidneys, ureters and bladder. Unenhanced (no oral or intravenous contrast) CT using $5 \mathrm{~mm}$ contiguous sections through the renal calculus was performed using a soft tissue setting of a window width and level of 280 and 15 Hounsfield units respectively, on a Siemens Somatom Emotion 6 slice scanner (Siemens Medical Solutions, Munich, Germany) at 110 - 140 kv, $72-$ $90 \mathrm{~mA}$ and scan time 10-15 seconds.

The image showing the calculus in largest dimension was selected and a pixel map of the largest possible region of interest with in the calculus was generated. The mean calculus attenuation value was calculated. Patients were divided into two groups (A and B), depending on the CT attenuation values of less or more than $750 \mathrm{HU}$ s respectively.

All treatment was done by an electromagnetic lithotripter (Dornier Compact Delta, Singapore). Calculi were fragmented under fluoroscopic guidance. A change in

\begin{tabular}{|l|l|}
\hline Stone location & Number of subjects (percentage) \\
\hline Renal pelvis & $10(33 \%)$ \\
\hline Upper calyx & $6(20 \%)$ \\
\hline Mid-calyx & $5(16.6 \%)$ \\
\hline Lower calyx & $2(6.6 \%)$ \\
\hline Upper ureter & $7(23.3 \%)$ \\
\hline \multicolumn{2}{|c|}{ Table-1: Showing distribution of subjects with respect of } \\
location of stone
\end{tabular}

calculus size and/or outline, or separation of stone fragments indicated fragmentation. During 1 ESWL session a maximum of 3,000 shocks were delivered at the energy level of 2 to 4 , corresponding to 14 to $15 \mathrm{kV}$.

Treatment was terminated if satisfactory fragmentation was noted earlier. Another ESWL session was done after 6 weeks if follow-up plain x-ray of the kidneys, ureters and bladder showed significant residual fragments. When a calculus did not fragment ever after 3 sessions, the patient was offered alternative therapy. The total number of shock waves delivered to any renal unit were limited to a maximum of 9000 during the course of treatment.

Plain x-ray of the kidneys, ureters and bladder was performed 6 weeks after the completion of treatment for assessing the outcome. Cases of residual fragments larger than $5 \mathrm{~mm}$ or of a stone that did not fragment satisfactorily were considered failures. Mean HU per stone was compared in the stone free and residual stone groups.

\section{STATISTICAL ANALYSIS}

Statistical analysis was performed using student $t$ test using statistical software StatPac version 4.0 (StatPac, Inc., Bloomington, $\mathrm{MN}$ ). $\mathrm{p}$ value of $<0.05$ was considered to be statistically significant.

\section{RESULTS}

A total of 30 patients were studied who met the study inclusion criteria, none of the patient lost to follow-up. Out of these, 21 were males and 9 were females with mean age of 35.43 years.

A total of 7 stones $(23.3 \%)$ were located in the ureter. A total of 23 stones (76.6\%) were located in the kidney (Table 1$)$. Of the intra-renal calculi 10 (33.3\%), 6 (20\%), 5 (16.6\%) and $2(6.6 \%)$ were in the renal pelvis, upper pole, mid pole and lower pole, respectively. The mean number of shock waves delivered in two groups A and B were 4411.76 and 7615.38 respectively $(\mathrm{p}<0.0001)$.

Out of these, 17 belonged to group A (stone HU value $<750 \mathrm{HU}$ ) and 13 belonged to group B (stone attenuation value $\geq 750 \mathrm{HU}$ ). There was no significant difference in size of stones in two groups. Mean stone size in group A was $1.51 \mathrm{~cm}$ and that in group B was 1.59 ( $\mathrm{p}$ value- 0.5346 ) The mean number of shock waves delivered in two groups $A$ and $B$ were 4411.76 and 7615.38 respectively $(p<0.001)$. The stone clearance rate in Group A was $100 \%$ versus Group B where it was only $15.4 \%$ ( $p<0.001)$. Out of 11 subjects with treatment failure in group $B$, two had inadequate fragmentation with significant residual stone fragments in urinary system while 9 subjects showed no fragmentation on ESWL (Table 2).

\begin{tabular}{|l|c|c|c|c|c|c|c|}
\hline Group & $\begin{array}{c}\text { No. of } \\
\text { subjects }\end{array}$ & $\begin{array}{c}\text { Mean no. of } \\
\text { shock waves } \\
\text { delivered }\end{array}$ & $\begin{array}{c}\text { Mean no. of } \\
\text { ESWL sessions }\end{array}$ & $\begin{array}{c}\text { Mean stone } \\
\text { size }\end{array}$ & $\begin{array}{c}\text { Fragmentation } \\
\text { percentage }\end{array}$ & $\begin{array}{c}\text { Significant } \\
\text { residual } \\
\text { fragments }\end{array}$ & $\begin{array}{c}\text { No } \\
\text { fragmentation }\end{array}$ \\
\hline Group A & 17 & 4411.76 & 1.47 & $1.51 \mathrm{~cm}$ & $17(100 \%)$ & 0 & 0 \\
\hline Group B & 13 & 7615.38 & 2.46 & $1.59 \mathrm{~cm}$ & $2(15.4 \%)$ & 2 & 9 \\
\hline$p$ value & $<0.001$ & $<0.01$ & $>0.05$ & $<0.001$ & & \\
\hline \multicolumn{7}{|l|}{ Table-2: Showing average stone size, shock waves delivered and stone clearance rate in two groups } \\
\hline
\end{tabular}


Overall, mean attenuation values for the stone-free and residual stone groups were significantly different (514.10 versus $970.36 \mathrm{HU}$, respectively, $\mathrm{p}<0.0001$ ).

Two patients developed hematuria after lithotripsy sessions and were managed expectantly.

\section{DISCUSSION}

Kidney stones are a significant source of morbidity. $80 \%$ of those with kidney stones are men; most stones in women are due to either metabolic defects (such as cystinuria) or infection. Men most commonly experience their first episode between ages 30-40 years, while for women the age at first presentation is somewhat later. Recurrence rates are estimated at $50 \%$ over a 10 years period and $75 \%$ over 20 years periods with some experiencing ten or more episodes over the course of a lifetime. ${ }^{5}$

In India, more than 5 million patients suffer from urinary stone disease and at least $1 / 1000$ of them need hospitalization at some time in their life for its management. ${ }^{6}$ The recurrence of stone disease is higher in males compared to female. It is thought to be due to lithogenic effect of testosterone and lack of inhibiting effect of estrogen hormone. ${ }^{7}$

In India, Maharashtra, Gujarat, Punjab, Haryana, Delhi and Rajasthan are the regions most affected by urinary stone disease. Surgery for removal of urinary stones forms one of the commonest operations in hospitals in these regions. ${ }^{8}$

NCCT can be rapidly performed even in unstable patients and those with renal impairment. It provides an abundance of information on urinary tract calculi, including size, shape, number and location. NCCT can detect even almost all radiolucent stones which can be missed on conventional radiographs. In addition, the attenuation value of calculi measured in $\mathrm{HU}$ obtained from non-contrast $\mathrm{CT}$ may be used to predict stone composition. ${ }^{4}, 9,10$

In present study, $70 \%$ of the subjects with stone disease were males which is consistent with other studies in literature. ${ }^{4,11,12}$ A higher incidence in male subjects can be explained by due to hormonal differences and relative lack of stone inhibitors in males in reproductive age group. ${ }^{13}$

This study provides compelling data suggesting the importance of measuring $\mathrm{HU}$ in all patients who undergo non-contrast $\mathrm{CT}$ to evaluate urinary calculi. By evaluating patients undergoing ESWL for upper urinary tract calculi we determined whether the success of this procedure could be predicted by pretreatment $\mathrm{HU}$ values measured on noncontrast CT. Furthermore, we included only patients with a single stone between 5 and $20 \mathrm{~mm}$ which allowed for the evaluation of a homogeneous group of stones commonly treated with ESWL. Inclusion of all stones of various sizes would have diluted the data, making it uninterpretable. Because of fairly uniform groups, there was no significant difference in stone size between two groups with different HU values.

In the present study, when patients were categorized by calculus density, $56.66 \%$ with calculi of $<750 \mathrm{HU}$ had complete clearance with $100 \%$ success rate. Conversely, of patients with calculi of $\geq 750 \mathrm{HU}$ ( $43.34 \%$ subjects), only $15.4 \%$ could clear the stones completely, leaving rest as partially fragmented or un-fragmented. Analysis indicated that the attenuation value (calculus density) had an inverse relation with outcome. In the study of Omer et $\mathrm{al},{ }^{14}$ the rate of stone clearance in upper urinary system using ESWL was 100\% with stones of $<500 \mathrm{HU}$ value while those with $>1000 \mathrm{HU}$ had only $10 \%$ clearance rate. While in the study of Naik et $\mathrm{al},{ }^{4}$ the success rate was $100 \%$ with stones of $<500 \mathrm{HU}$ value and about $33 \%$ for $>1000 \mathrm{HU}$ stones. In the study of Joseph et $\mathrm{al}^{16}{ }^{16}$ the success rate was $100 \%$ with stones of $<500 \mathrm{HU}$ value and about $55 \%$ for $>1000 \mathrm{HU}$ stones. Therefore, all these studies demonstrate $100 \%$ success rate with stones of $<500 \mathrm{HU}$, while the success rate was low for stones more than $1000 \mathrm{HU}$. In our study, the success rate was $100 \%$ for stones even upto $750 \mathrm{HU}$ value with markedly decreased success rate of ESWL above this value. In the study of Gupta et $\mathrm{al},{ }^{16}$ the success rate was $88 \%$ for stones more than $750 \mathrm{HU}$ while it was $65 \%$ for stones more than $750 \mathrm{HU}$. There was significant difference in mean attenuation value in stone-free and residual stone groups ( 514.10 versus $970.36 \mathrm{HU}$ ). Pareek et $\mathrm{al}^{17}$ observed similar findings. In their study, the mean HU value of stone free group was significantly lower than that with residual stone group (551HU vs $926 \mathrm{HU})$. Ouzaid et al ${ }^{18}$ suggested a threshold of $970 \mathrm{Hu}$ for predicting success of ESWL in stone clearance. Thus, results of present study are in agreement with previous studies and in reiterates the fact that stone attenuation value has an inverse relation with success rate of ESWL.

In present study, there is no statistically significant difference in size of stones in groups with treatment success and failure. Similar observations have been made by other authors., ${ }^{4,18}$

Mean number of ESWL sessions required was significant higher in group with stones of $>750 \mathrm{HU}$ value (1.47 vs 2.46 ), which means that stones with higher attenuation value require more number of ESWL sessions. Similar observations have been made in other studies. ${ }^{4,19}$

\section{CONCLUSION}

In conclusion, Patients with $\mathrm{HU}<750$ have higher significantly stone fragmentation rate (100\%) versus patients with $\mathrm{HU}>750$ (15.4\%), which suggests that the HU measurement of upper urinary calculi (renal or upper ureteric) on pre-treatment non-contrast $\mathrm{CT}$ predicts the stone-free rate after ESWL. HU determination on noncontrast CT as well as stone size may be beneficial for selecting the preferred treatment option, that is ESWL, ureteroscopy or percutaneous nephro-lithotomy for patients with urinary calculi.

\section{REFERENCES}

1. Chaussy C, Brendel W, Schmiedt E. Extracorporeally induced destruction of kidney stones by shock waves. Lancet 1980;2(8207):1265-8.

2. Johnson EK, Faerber GJ, Roberts WW et al. Are stone protocol computed tomography scans mandatory for children with suspected urinary calculi? Urology 2011;78(3):662-6.

3. Welk BK, Teichman JMH. Uric acid nephrolithias in the era of noncontrast computed tomography. Canadian Urological Association Journal 2008;2(4):420-1.

4. Naik D, Jain A, Hedge AA, Kumar AA. Determination 
of Attenuation Values of Urinary Calculi by NonContrast Computed Tomography and Correlation with Outcome of Extracorporeal Shock Wave Lithotripsy A Prospective Study. International Journal of Anatomy, Radiology and Surgery 2017;6(2):RO81-RO86.

5. Weiss M, Liapis H, Tomaszewski JE and Arend LJ, Chapter 22: Pyelonephritis and other infections, reflux nephropathy, hydronephrosis, and nephrolithiasis, pp. 991-1082 in Jennette and Heptinstall (2007).

6. Sangeetha M, Gayathiri K, Sharanya VK. Phytoconstituents with antiurolithic activity-a review. International Journal of Biological and Pharmaceutical Research 2015; 6(12):1001-7.

7. Devi VK, Baskar R, Varalakshmi P. Biochemical effects in normal and stone forming rats treated with the ripe kernel juice of plantain (musa paradisiaca). Ancient Science of Life 1993;12(3-4):451-61.

8. Gindodia KR, Thakare S, Manekar A. Comparitive study between allopathic medicines and ayurvedic herbal medicines in treatment of nephrolithiasis and ureterolithiasis. Paripex - Indian journal of research 2017;6(8):15-6

9. Mostafavi MR, Ernst RD, Saltzman B. Accurate determination of chemical composition of urinary calculi by spiral computerized tomography. J Urol 1998;159(3):673-5.

10. Saw KC, McAteer JA, Fineberg NS, et al. Calcium stone fragility is predicted by helical CT attenuation values. J Endourol. 2000;14(6):471-4.

11. Tanaka M, Yokota E, Toynaga Y, Shimizu F, Ishii $\mathrm{Y}$, Fujime $\mathrm{M}$ et al. Stone attenuation value and cross sectional area on $\mathrm{CT}$ predict the success of shock wave lithotripsy. Korean J Urol 2013; 54(7):454-9.

12. Dahiphale D, Apte A, Dahiphale AP. Non-contrast spiral computed tomography diagnosis of urolithiasis and associated features: hospital based study. Int J Res Med Sci 2016;4(10):4286-9.

13. Younis A, Radharikrishnan S. Kidney stone disease. Trends in Urology and Men's Health 2011;2(1):15-20.

14. Omer MAE, Abdelazim HK, Faisal YA, Tarig HHA. Value of Hounsfield Unit in Prediction of Stone Free Rate in Management of Upper Urinary Tract Stones Using $\mathrm{Ct}$ and Eswl. JOJ uro and nephron 2017;2(5):555600.

15. Joseph P, Mandal AK, Singh SK, Mandal P, Sankhwar SN, Sharma SK. Computerized tomography attenuation value of renal calculus: can it predict successful fragmentation of the calculus by extracorporeal shock wave lithotripsy? A preliminary study. J Urol 2002;167(5):1968-71.

16. Gupta NP, Ansari MS, Kesarvani P, Kapoor A, Mukhopadhyay S. Role of computed tomography with no contrast medium enhancement in predicting the outcome of extracorporeal shock wave lithotripsy for urinary calculi. BJU Int 2005;95(9):1285-8.

17. Pareek G, Armenakas NA, Fracchia JA. Hounsfield units on computerized tomography predict stone-free rates after extracorporeal shock wave lithotripsy. J Urol 2003;169(5):1679-81.

18. Ouzaid I, Al-qahtani S, Dominique S, Hupertan V, Fernandez P, Hermieu JF, et al. A 970 Hounsfield units
(HU) threshold of kidney stone density on non-contrast computed tomography (NCCT) improves patient selection for extracorporeal shock wave lithotripsy (ESWL): evidence from prospective study. BJU Int 2012;110(11 Pt B):E438-42.

19. Massoud AM, Abdelbary AM, Al-Dessoukey AA, Moussa AS, Zayed AS, Mahmmoud O. The success of extracorporeal shockwave lithotripsy based on the stone attenuation value from noncontrast computed tomography. Arab J Urol 2014;12(2):155.

\section{Source of Support: Nil; Conflict of Interest: None}

Submitted: 13-04-2018; Accepted: 12-05-2018; Published online: 22-05-2018 